\title{
Delayed Intracerebral Hemorrhage from a Pseudoaneurysm Following a Depressed Skull Fracture
}

\author{
Pouya Nazari, MD, Manish K. Kasliwal, MD, MCh, Joshua T. Wewel., MD, \\ Sumeet G. Dua*, MD \& Michael Chen, MD
}

A 26-year-old male presented with delayed intracerebral hemorrhage from a ruptured distal middle cerebral artery pseudoaneurysm that followed a compound depressed skull fracture from years ago. The brain protrusion through the skull defect likely resulted in stretching and subsequent tearing of the arterial wall resulting in the pseudoaneurysm formation. No prior report of such a clinical occurrence exists in the literature. We highlight an unusual but treatable cause for intracerebral hemorrhage following surgery for traumatic brain injury.

Key Words: Middle cerebral artery; Pseudoaneurysm; Depressed skull fracture; Treatment; Delayed; Hemorrhage

Depressed cranial fractures may complicate up to $6 \%$ of head injuries and account for significant morbidity and mortality [1]. Common complications from depressed skull fractures include infection, epilepsy, neurological deficits and mortality. Surgical treatment of compound depressed cranial fractures often involves debridement and elevation to decrease the incidence of infection. While not seen in teenagers or adults, a

All authors: Department of Neurosurgery \& ${ }^{*}$ Radiology, RUSH University Medical Center, Chicago, IL-60612, USA

Received November 21, 2015;

accepted after revision January 20, 2016.

Correspondence to: Manish K. Kasliwal, MD, MCh

Department of Neurosurgery, Suite 855, RUSH University Medical Center, Chicago, IL-60612, USA

Tel. 312.942.6644 Fax. 312.942.2176

E-mail: manish_kasliwal@rush.edu

This is an Open Access article distributed under the terms of the Creative Commons Attribution Non-Commercial License (http://creativecommons.org/licenses/by-nc/3.0) which permits unrestricted non-commercial use, distribution, and reproduction in any medium, provided the original work is properly cited. growing skull fracture is a rare complication of skull fractures in children usually less than three years of age. A hypothesized pathophysiological mechanism involves herniation of the leptomeninges with rupture of the dura during the skull fracture [2]. We encountered a rare case of intracranial hemorrhage secondary to a de novo distal middle cerebral artery (MCA) pseudoaneurysm within the exact location of an incompletely healed depressed skull fracture. The likely pathogenesis may have been the protrusion of brain parenchyma with associated vasculature through dural laceration sustained during initial brain injury and subsequent trauma from the incompletely healed bony defect between the skull and craniotomy. To our knowledge, there is no prior report of a traumatic pseudoaneurysm following a depressed skull fracture in the literature.

\section{CASE REPORT}

A 26-year-old male presented to us following a 
seizure with left sided weakness (4-/5, Medical Research Council (MRC) grade). The past medical history was significant for a traumatic brain injury seven years prior that involved a depressed skull fracture after being hit with a baseball bat. Craniotomy and repair of the depressed skull fracture occurred at an outside institution. A brain computed tomography (CT) scan at admission showed a $3.5 \times 2.6 \mathrm{~cm}$ hematoma in the right parietal lobe adjacent to the prior craniotomy site. There was also bony separation at the margins of the prior craniotomy with presence of protrusion of the brain parenchyma through the defect (Fig. 1A, C). A magnetic resonance angiogram (MRA) of the brain suggested a pseudoaneurysm (Fig. 1D), which was confirmed on a catheter cerebral angiogram that demonstrated the presence of a pseudoaneurysm arising from the right middle cerebral artery M4 segment measuring $3 \times 3 \mathrm{~mm}$ in size with a neck of $1 \mathrm{~mm}$ that was successfully coiled (Fig. 2A, D). An excelsior SL-10 micro catheter (Stryker, Kalamazoo, Michigan, U.S.A.) was used for navigating the vascular anatomy, followed by deployment of a single platinum coil measuring $2.5 \times 5$ to achieve complete occlusion of the aneurysm. A new occlusion of the distal branches of the angular artery, likely thromboembolic, was identified on the final angiogram, but was
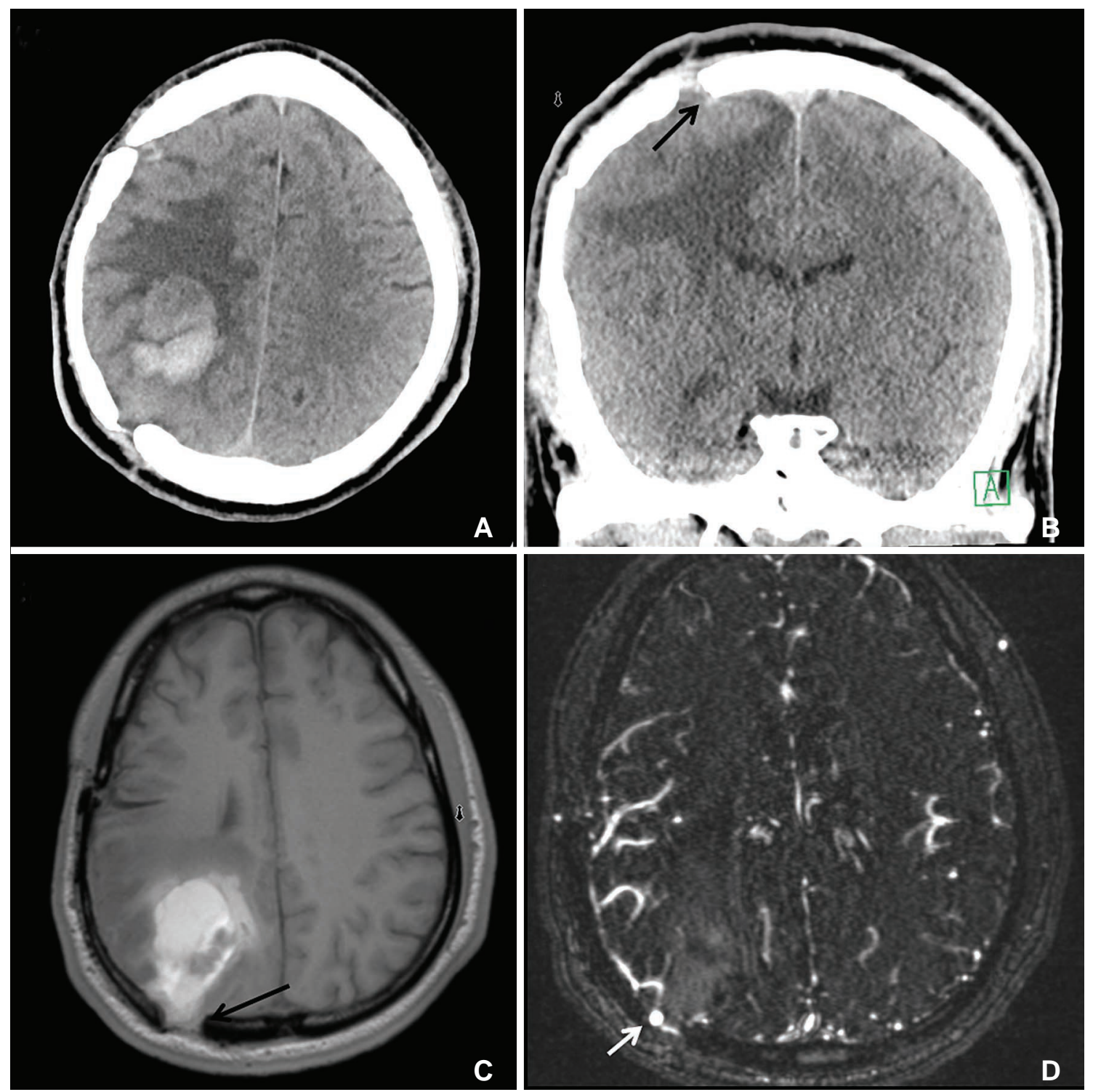

Fig. 1. Axial non contrast CT scan of the head $(\mathbf{A}$ and $\mathbf{B})$ and $M R I(C)$ showing a $3.5 \times 2.6 \mathrm{~cm}$ acute intraparenchymal hematoma in the right parietal lobe along with a large defect in the cranium in the parietotemporal region from prior craniotomy. An old, right parietal craniotomy is redemonstrated. Also visible is the protrusion of the brain parenchyma through the defect (arrows in the B and C), particularly along its superior aspect seen both on CT and axial MRI. An axial MRA image (D), suggestive of a pseudoaneurysm (arrow). 


\section{Pouya Nazari, et al.}

regarded as clinically insignificant (Fig. 2C, D).

The post-operative course was uneventful with improvement in left-sided hemiparesis to almost normal strength, and the patient was discharged home on day three. He had been doing well at the 12-month follow-up, with no motor or sensory deficits and a modified Rankin scale score of 1 . The patient does have occasional partial seizures likely from the prior depressed skull fractures (Engle class Ib), which are currently well-controlled with the use of medications.

\section{DISCUSSION}

Intracranial aneurysms can be classified as congeni- tal, atherosclerotic, dissecting, infectious, and post traumatic [3]. Traumatic intracranial aneurysms (TICAs) are rare and account for less than $1 \%$ of the intracranial aneurysms and are more common in children and adolescents. They can occur after even mild or seemingly trivial head trauma, and are associated with a morbidity and mortality rate as high as $50 \%$. The MCA has the most complex branching pattern of the major intracranial arteries and TICAs are frequently found in the cortical branches of middle cerebral artery (MCA) [4-6]. TICAs may develop beneath an overlying linear or depressed skull fracture and are attributed to direct vessel trauma leading to formation of a pseudoaneurysm.
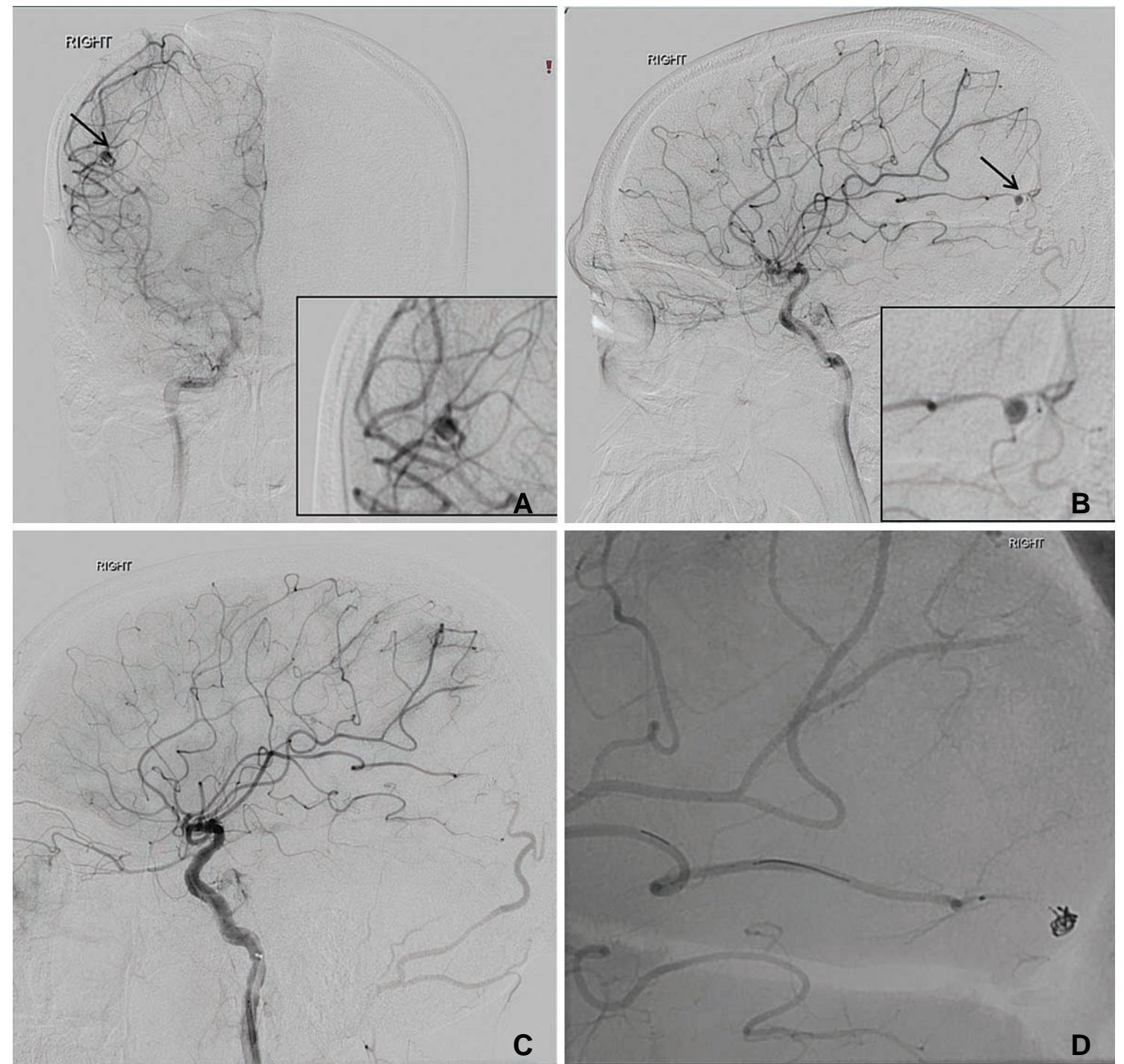

Fig. 2. AP $(\mathbf{A})$ and lateral $(\mathbf{B})$ views of conventional angiograms following injection of the right-sided internal carotid artery, showing the pseudoaneurysm (arrows) arising from the right middle cerebral artery M4 segment, measuring $3 \times 3 \mathrm{~mm}$ in size with a neck of $1 \mathrm{~mm}$ (2A and B, middle image) that was successfully coiled (2C). Insets in $\mathbf{2 A}$ and $\mathbf{B}$ show a magnified view of the aneurysm. An unsubtracted lateral view (D) of the angiogram showing coil mass occluding the aneurysm. 


\section{Delayed Pseudoaneurysm after Cranial Trauma}

Histologically, TICAs can be classified as true, false, dissecting or mixed based on the damaged structures of the vascular wall $[4,5,7]$. A pseudoaneurysm (false aneurysm), which is the most common type, occurs when all layers of the arterial wall are lacerated, and is almost always associated with direct injury to cortical arteries, which are often beneath a skull fracture. Several mechanisms have been proposed in the formation of traumatic aneurysms, all of which involve either direct injury to the vessel or stretching of the vessel by adjacent forces $[6,8,9]$. The case we encountered is very unusual, as the aneurysm did not develop from the direct trauma of the vessel from the depressed bone fragments; but rather occurred secondary to the outpouching of the brain parenchyma through the dural defect between the edges of the craniotomy flap and skull made during the initial repair of the depressed skull fracture (Fig. 1). This likely resulted in stretching and tearing of the arterial wall from the bony edges leading to formation of a pseudoaneurysm.

While debridement, elevation of the fracture, removal of bone fragments, and delayed cranioplasty (if needed) remains the most common way to manage depressed skull fractures, there is an increasing consensus that immediate bone fragment replacement/cranioplasty does not result in any increase in infectious complications and may be preferable as it also obviates the need for a second procedure $[1,10]$. Also, while prevention of infection and epilepsy remains the main driver of treatment for a compound depressed fracture, the present case highlights an unusual complication of a traumatic pseudoaneurysm formation that can be a cause of delayed morbidity and perhaps mortality. As the pseudoaneurysm likely resulted from the herniation of brain parenchyma through the dural defect, the likely pathogenesis involved presence of an occult dural tear that was sustained at the time of initial injury and was not apparent during the initial surgery.

\section{CONCLUSION}

A rare of case of a delayed traumatic pseudoaneurysm of the middle cerebral artery following surgical repair of a compound depressed fracture of the skull is reported. Being aware of this complication in providing long-term treatment to patients with depressed skull fractures can be helpful to avoid and treat this unusual complication.

\section{References}

1. Bullock MR, Chesnut R, Ghajar J, Gordon D, Hartl R, Newell DW, et al. Surgical management of depressed cranial fractures. Neurosurgery 2006;58:S56-60

2. Prasad GL, Gupta DK, Mahapatra AK, Borkar SA, Sharma BS. Surgical results of growing skull fractures in children: a single centre study of 43 cases. Childs Nerv Syst 2015;31:269-277

3. Ohta M, Matsuno H. Proximal M2 false aneurysm after head trauma--Case report. Neurol Med Chir (Tokyo) 2001;41:131-134

4. Yi HJ, Kim KM, Ko Y, Kim YS, Oh SJ, Oh SH. A spontaneous giant pseudoaneurysm presenting with chronic headache in adolescent. Childs Nerv Syst 2006;22:295-299

5. Voelker JL, Ortiz O. Delayed deterioration after head trauma due to traumatic aneurysm. $W$ V Med J 1997;93:317-319

6. Larson PS, Reisner A, Morassutti DJ, Abdulhadi B, Harpring JE. Traumatic intracranial aneurysms. Neurosurg Focus 2000;8:e4

7. Komiyama M, Morikawa T, Nakajima H, Yasui T, Kan M. "Early" apoplexy due to traumatic intracranial aneurysm--case report. Neurol Med Chir (Tokyo) 2001;41:264-270

8. Miley JT, Rodriguez GJ, Qureshi AI. Traumatic Intracranial Aneurysm Formation following Closed Head Injury. J Vasc Interv Neurol 2008;1:79-82

9. Fleischer AS, Patton JM, Tindall GT. Cerebral aneurysms of traumatic origin. Surg Neurol 1975;4:233-239

10. Wylen EL, Willis BK, Nanda A. Infection rate with replacement of bone fragment in compound depressed skull fractures. Surg Neurol 1999;51:452-457 